\title{
Improvement of Musculoskeletal Model inputs: Adjustment of Acceleration by Dynamic Optimization
}

\author{
F. LEBOEUF* $*$, F. COLLOUD $\ddagger$ \\ $†$ CHU de Nantes, pôle Médecine Physique et Réadaptation, Nantes, 44096 France \\ \$Institut Pprime UPR 3346 CNRS - Université de Poitiers,Poitiers,86962 Futuroscope \\ Keywords: Acceleration, Dynamic optimization, Inverse dynamics, Muscle tension, Static optimization.
}

\section{Introduction}

The knowledge of intrinsic dynamics in terms of joint torques and muscle tensions is of importance for clinical investigations. The common process is to solve a multibody inverse dynamic problem based on a set of iterative equations using noisy experimental data as guest. Body segment accelerations are usually assessed by double differentiation, a method well-known to amplify kinematic measurement noise. As a result, iterative equations propagate uncertainties leading to inconsistencies between measured external force and the rate of change of linear momentum.

Recent studies addressed this residual force problem by adjusting mass distribution while they calculate force tensions [1] or by dealing with acceleration computation [2,3]. However, these different approaches were based on a least-square problem still leading to approximate intrinsic dynamics.

The aim of this communication is to compute joint accelerations by solving a dynamic optimization problem. We will examine the effect of the optimal adjustment on joint torques and muscle tensions.

\section{Methods}

\section{Acceleration adjustments}

The optimization problem consisted in minimizing the sthenic criterion:

$$
J=\sum_{t=t_{1}}^{T}\left(F\left(q_{i}(t), \dot{q}_{i}(t), \ddot{q}_{l}(t)\right)-F^{m}(t)\right)^{2}
$$

while respecting the dynamic model:

$$
\tau_{i}(t)=B\left(q_{i}(t), \dot{q}_{i}(t), \ddot{q}_{l}(t)\right)
$$

where $q_{i}(t)$ is the vector of absolute joint coordinates (for $i=1, \ldots, 4$ ), $\dot{q}_{i}$ and $\ddot{q}_{l}$ are the first and second order time-derivatives of $q_{i}$, $F\left(q_{i}(t), \dot{q}_{i}(t), \ddot{q}_{l}(t)\right)$ and $F^{m}(t)$ are the vectors of ground reaction force expressed as a function of the measured joint and measured by a force platform and $\tau_{i}$ is the vector of joint driving torques.

This dynamic problem is recasted in a parametric optimization problem [4] through the use of a quartic spline of class $C^{2}$. Over each subintervals $I_{j}=\left[t_{j}, t_{j+1}\right]$, the forth-order polynom is defined: For $j \leq n$,

$$
t \in I_{j}, \alpha=t_{j+1}-t_{j} \tau=\frac{t-t_{j}}{\alpha}
$$

$$
q_{i}(t) \approx c_{i j 0}+c_{i j 1} \tau+c_{i j 2} \tau^{2}+c_{i j 3} \tau^{3}+c_{i j 4} \tau^{4}
$$

Considering that at each connecting point, the joint coordinates $q_{i}$ derived from measurement. The link relations on the first and second derivatives let appear the coefficients as function of a vector $X^{i}$ to optimize:

$$
X^{i}=\left(\ddot{q}_{i}(0), \ldots, \ddot{q}_{i}\left(t_{j}\right), \ldots, \ddot{q}_{i}(T)\right)^{T}
$$

which collected the joint accelerations at each connecting points.

\section{Muscle tension assessment}

Muscle tensions of the knee flexors: long hamstrings $T_{1}$, short hamstrings $T_{2}$ and gastrocnemius $T_{3}$ were assessed by solving the quadratic optimization problem:

$$
\min \sum_{i=1}^{3}\left(\frac{T_{i}}{T_{\max , i}}\right)^{2}
$$

subjected to linear equality constraints:

$$
\sum_{i=1}^{3} r_{i} T_{i}=\tau_{\text {knee }}
$$

and inequality constraints:

$$
0 \leq T_{i} / T_{\max } \leq 1
$$

The muscle properties $\left(r_{i}\right)$ were taken from [5]

\section{Experimental data and inverse dynamics}

A subject (mass: $76 \mathrm{Kg}$, height: $1.76 \mathrm{~m}$ ) performed at self-selected velocity a knee flexion on a force plate (Kistler, Switzerland) sampled at $1000 \mathrm{~Hz}$. The tridimensional trajectories of 16 markers were simulnateously recorded at $50 \mathrm{~Hz}$ using a motion analysis system (Biogesta, France). Joint torques $\left(\tau_{i}\right)$ of a 4-segment planar model were computed by a top-down process based on iterative NewtonEuler equations in which inertial parameters were provided by de Leva [6].

\section{Analysis of results}

Numerical optimizations were tested on the knee flexion with 51 connecting points. Our optimal inverse dynamic approach was compared with classical top-down inverse dynamics in terms of ground reaction forces, joint accelerations, joint torques and muscle tensions by computing root mean square differences (RMSd). 


\section{Results and Discussion}

Dynamic optimization was effective. The unknown joint accelerations were perfectly adjusted to match the discrete force plate measurements. No residual force (table 1) were observed

Table 1: RMSd with measured groud reaction force as reference.

\begin{tabular}{c|cc} 
Forces & Optimal & Non-optimal \\
\hline Horizontal & 0 & 24 \\
Vertical & 0 & 27
\end{tabular}

Consequently, joint accelerations, joint torques and muscle tensions (figure 1) gave a new insigth of the movement.

joint acceleration were underestimated with common double differentiation (non-optimal method) As an example, the knee acceleration amplitude was $60 \%$ larger while it was computed using our optimization approach (Fig. 1, top).

Regarding dynamic parameters, the non-optimal method overestimated them. With adjusted acceleration,we highlighted a phase (from 0 to $0.48 \mathrm{~s}$ ) were muscle tensions remain stables. This would be hidden by the uncertainty propagation associated with double differentation. A reliability study should confirm it.

Contrary to least-square approach [2,3], the perfect relation between kinematics and dynamics would enhance the identification of pathological dysfunction as well as the efficiency of forward simulation

Our method is subject to limitations. It does not consider as [2] the force measurement uncertainty. Comparison with least-square method would be carried out in future research. Moreover, optimal accelerations are still sensitive to the inertial data. Further study would be to associated with an optimal mass distribution algorithm.

\section{Conclusions}

In conclusion, our optimal inverse dynamic method improves the dynamic model by proposing input kinematic data in accordance with force measurement. This innovative method allowed computing joint accelerations without implement the double differentiation. . Improving joint torques and thus muscle tension computation should enhance the dynamic simulation of musculoskeletal model.

\section{References}

[1] Riemer R. and Hsiao-Wecksler E., Improving net joint torque calculations through a two-step optimization method for estimating body segment parameters, J. Biomech. Eng., 2009, Vol. 131(1).

[2] Cahouët V., Martin L. and Amarantini D., Static optimal estimation of joint accelerations for inverse dynamics problem solution, J. Biomech., 2002, Vol. 35(11), 1507-13

[3] Kuo A., A least-squares estimation approach to improving the precision of inverse dynamics computations, J. Biomech. Eng., 1998, Vol. 120, 148-59.

[4] G. Bessonnet, P. Seguin, Generating optimal walking cycles using spline-based stateparameterization, International Journal of Humanoid Robotics (IJHR), vol. 2, No. 1, pp. 47-80, 2005

[5] Dul, J., Townsend, M.A., Shiavi, R., Johnson, G.E., 1984. Muscular synergism-I. On criteria for load sharing between synergistic muscles. Journal of Biomechanics 17 (9), 663-673

[6] De Leva, P., Adjustments to ZatsiorskySeluyanov's segment inertia parameters. Journal of biomechanics, 1996, Vol. 29(9), 1223-1230

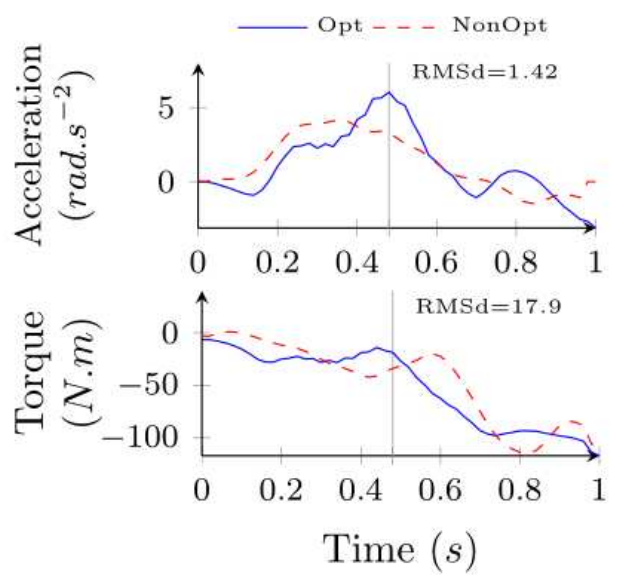

Long Harmstring
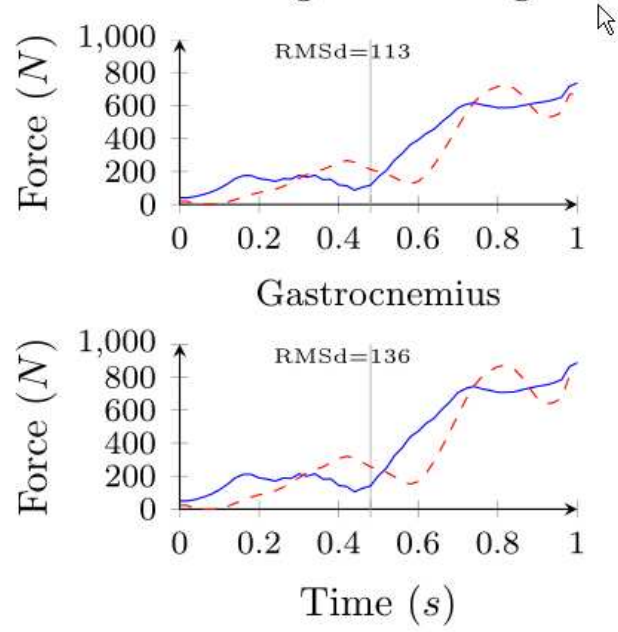

Figure 1: Knee acceleration (top), knee torque (middle) and muscle tensions (bottom) computed using non-optimal inverse dynamics and optimal approaches. 